\title{
The effect of scheduled antibody testing on treatment patterns in interferon-treated patients with multiple sclerosis
}

\author{
Edward Fox ${ }^{1 *}$, Barbara Green², Clyde Markowitz ${ }^{3}$, Ronald Murray ${ }^{4}$, Andrew D Goodman ${ }^{5}$, Stephen J Glenski ${ }^{6}$, \\ Pippa Loupe ${ }^{6}$ and Jo Nita Cogburn ${ }^{6}$
}

\begin{abstract}
Background: Many patients with relapsing-remitting multiple sclerosis (MS) treated with high-dose interferon- $\beta$ (IFN $\beta$ ) develop serum binding antibodies (BAb) and neutralizing antibodies (NAb). NAb reduces the biological activity of IFN $\beta$, which contributes to clinical failure in these patients. We investigated whether access to antibody (Ab) test results would alter usual care of (IFNß)-treated patients and whether BAb could predict NAb.

Methods: This was a randomized, controlled, open-label, parallel-group, multicenter study in patients with multiple sclerosis. Subjects ( $n=1358)$ were randomly assigned to Ab testing or usual care. BAb and NAb titres were measured using standard assays. Primary and secondary outcomes were the proportion of patients whose IFN $\beta$ therapy changed and the type of and reasons for therapy changes.

Results: Therapy changes differed between the Ab testing and usual care arms (19.6\% and $14.0 \%$, respectively; $p=0 \cdot 004$ ). Results from Ab testing were more frequently reported as the reason for therapy change in the $A b$ testing arm than in the usual care arm $(p<0.0001)$. NAb and BAb positivity significantly increased the likelihood of therapy change and reduced IFN $\beta$-associated adverse events. BAb titres were a significant predictor of NAb positivity $(p=0.0012)$. Initial BAb-positive and NAb-positive status in both study arms had a significant impact on the overall number of patients with a therapy change $(p<0.05)$.
\end{abstract}

Conclusion: Access to Ab test results impacted therapy management. BAb titres can predict NAb positivity in patients on high-dose IFN $\beta$.

Keywords: Antibody testing, Interferon- $\beta$, Neutralizing antibodies, Serum binding antibodies

\section{Background}

Many patients with relapsing-remitting multiple sclerosis (MS) treated with high-dose interferon- $\beta$ (IFN $\beta$ ) develop serum binding antibodies (BAb) and neutralizing antibodies $(\mathrm{NAb})$. Frequencies and titres of these antibodies may vary by IFN $\beta$ formulation and frequency and route of administration [1].

BAb bind to IFN $\beta$ but do not necessarily inhibit its biological activity, and they can be detected within the first month of therapy [2]. Approximately $50 \%$ of patients who develop BAb also develop NAb [3], and most

\footnotetext{
*Correspondence: foxtexms@gmail.com

${ }^{1}$ Central Texas Neurology Consultants, 16040 Park Valley Drive, Suite 100, Round Rock, TX 78681, USA

Full list of author information is available at the end of the article
}

patients who become NAb-positive $(+)$ do so within 2 years of starting IFN $\beta$ treatment [2]. More than $40 \%$ of patients treated with high-dose preparations of IFN develop NAb [1], which reduce the biological activity of IFN $\beta$, thus contributing to clinical failure [2,4]. Indeed, $\mathrm{NAb}(+)$ patients tend to have a higher annual relapse rate and an increase in active lesions as measured by magnetic resonance imaging (MRI) [2,5-11]. The appearance of $\mathrm{NAb}(+)$ titres precedes these outcomes [2].

The methodology to detect NAb is cumbersome and non-standardized; thus, simpler BAb assays are preferred for screening before analyzing for NAb. BAb screening has low false-negative rates and high sensitivity/specificity. There have been opposing assessments of the importance of BAb and NAb testing relative to clinical 
management of IFN-treated patients, specifically in Europe (European Federation of Neurologic Societies [EFNS]), America (American Academy of Neurology), and Canada [3,8,12].

This study investigated whether antibody (Ab) testing and knowledge of Ab status affect the usual care of patients treated with high-dose IFN $\beta$. Moreover, because some guidelines (e.g., EFNS) advocate the use of BAb as a preliminary test, we explored whether BAb alone would be a potential guide for managing patients on IFN $\beta$. Specifically, we analyzed the correlation between $\mathrm{BAb}$ and NAb titres and the presence of BAb as a predictor for NAb.

\section{Methods}

\section{Study design}

A randomized, controlled, open-label, parallel-group, multicenter observational study (registration: NCT00336557) in patients with MS was conducted at 147 centers in the United States. The study followed the principles of the Declaration of Helsinki International Conference on Harmonisation guidelines on good clinical practices and all applicable laws and regulations.

Investigators or designees explained the study procedures, risks, and potential benefits, if any, to all patients. Patients reviewed the study instructions and informed consent form and were given the time and opportunity to have any questions concerning the conduct of the study answered to their satisfaction.

The primary outcome measure was the evaluation of differences in therapy/management between clinicians who were provided NAb data and those who were not during the 12-month follow-up period. The secondary outcome measure was an assessment of the type of and reasons for changes in IFN therapy/management. Exploratory outcome measures included the relationship between $\mathrm{BAb}$ and $\mathrm{NAb}$ results, therapy/management changes, and targeted events.

Patients $(\mathrm{N}=1358)$ on subcutaneously administered high-dose IFN therapy-IFN $\beta$-1b $(250 \mu \mathrm{g}$ on alternate days) and IFN $\beta$-1a (22 or $44 \mu \mathrm{g}$ three-times weekly [t.i.w.]) - were enrolled and randomly assigned to either $\mathrm{Ab}$ testing or usual care. Patients in the scheduled $\mathrm{Ab}$ testing arm had four study visits, at least two BAb and NAb tests over 12 months, and a final visit at 12 months. Those who had not completed 24 months of continuous therapy on the same IFN were offered an optional $\mathrm{BAb}+/-\mathrm{NAb}$ at the final visit. Subjects in the usual care arm were followed for 12 months under usual care conditions with $\mathrm{BAb}$ and NAb testing at the initial visit and optional testing at the final visit. Additional visits during the year were at the discretion of the clinicians and patients. Investigators were informed of $\mathrm{Ab}$ test results for patients in the Ab testing arm only.
All patients who underwent a blood draw for Ab testing at the initial and final clinic visit in either arm were included in the exploratory analysis. Unscheduled visits and blood draws were allowed in either arm at any point during the 12-month study period.

\section{Patients}

Patients enrolled were men or women aged $\geq 18$ years who had a diagnosis of MS and were on high-dose IFN therapy (dosed according to manufacturer labelling) for 12 months to $\leq 4$ years, with no more than 60 days of cumulative planned interruption of treatment. Patients were excluded if they had received oral or parenteral corticosteroid therapy within the 2 weeks before the initial visit; had been treated with immunoglobulin G (IgG) or plasmapheresis within the previous 6 months; were being treated with once-weekly intramuscular IFN $\beta$-1a, glatiramer acetate, or any immunosuppressant; or had been previously tested for NAb.

\section{Outcome measures}

Blood was drawn 48 hours after the last administration of IFN $\beta$ and at least 2 weeks after any use of systemic corticosteroids and sent to the Central Laboratory (FOCUS Bio-Inova, Cypress, CA) for analysis.

\section{$B A b$ assessment}

BAb assessments were conducted with a capture enzymelinked immunosorbent assay methodology to assess the presence of IgG antibodies that bind IFN $\beta$. A sample was considered $\mathrm{BAb}(+)$ if the result was $\geq 4 \cdot 0$ units.

\section{$N A b$ assessment}

Only the samples of patients who tested positive for $\mathrm{BAb}$ were analyzed for NAb positivity using the viral cytopathic effect assay method. Per laboratory standards, NAb values $<20$ units were considered negative $(\mathrm{NAb}[-])$, and values $\geq 20$ units were considered positive $(\mathrm{NAb}[+])$.

Physicians were notified of NAb results for $\mathrm{BAb}(+)$ patients in the Ab testing group only. All physicians and patients were notified of NAb and BAb status at the conclusion of the trial. BAb-negative (BAb[-]) samples were randomly assessed for NAb to ensure quality control.

\section{Management/therapy change questionnaire}

The investigator evaluated the patient for a change in clinical status and recorded on a questionnaire whether or not a change in management/therapy had occurred or was planned. Factors that had an impact on the decision to change management/therapy were also recorded. 


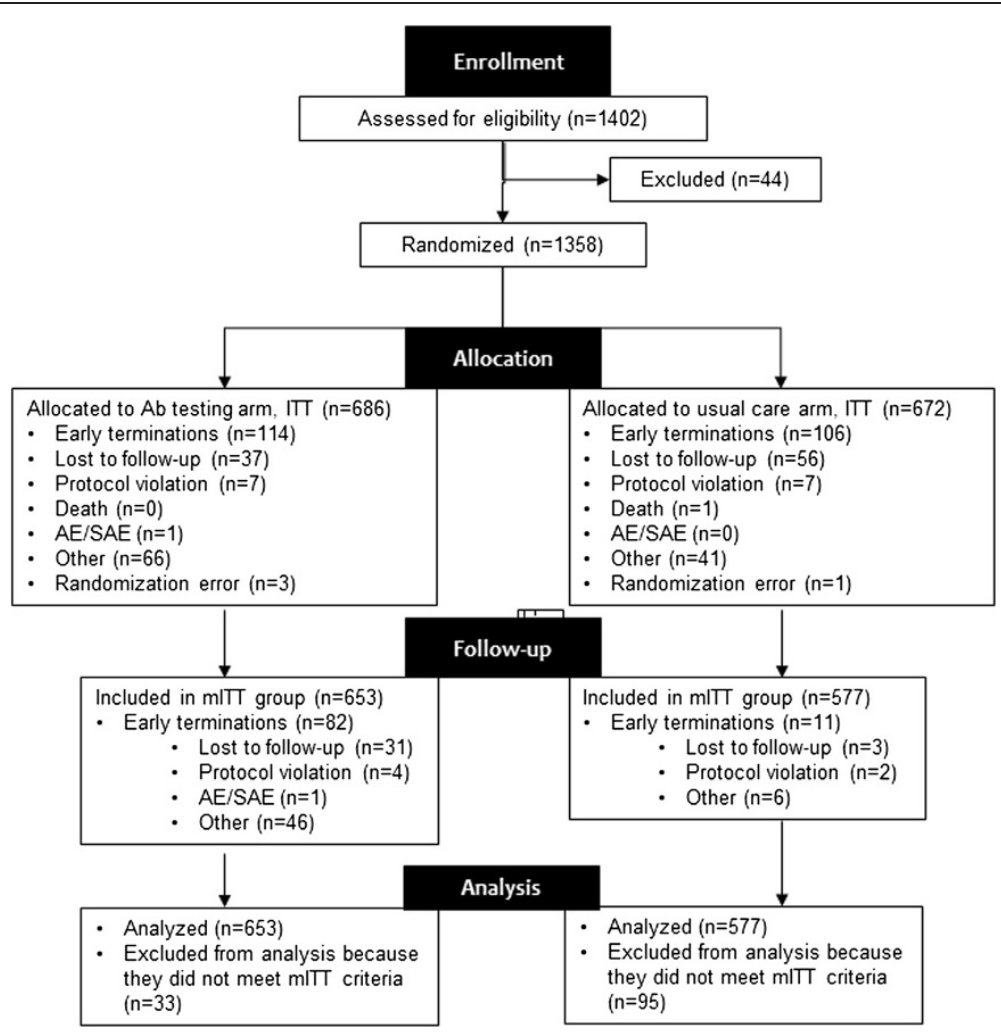

Figure 1 Patient disposition. Ab, antibody; AE, adverse event; ITT, intention-to-treat; mITT, modified ITT; SAE, serious adverse event.

\section{Safety measures}

The most common adverse events (AEs) known to be associated with high-dose IFN treatment were collected at each visit (targeted events): flu-like symptoms, local injection-site reactions (red, warm to the touch, painful, raised area around site), depression, seizures, menstrual irregularities, abnormal liver function tests (alanine aminotransferase and aspartate aminotransferase), cytopenias (lymphopenia, neutropenia, leukopenia, and thrombocytopenia), and decreased hemoglobin.

\section{Statistical analysis}

Patients were randomly assigned using a 1:1 assignment ratio and stratified by center using SAS $^{\circ}$ (SAS Institute Inc., Cary, NC: version 9.1) random number procedure. The sample size of 1350 patients provided $99.6 \%$ power for a therapy change rate of $4.4 \%$ in the usual care arm and an $11.2 \%$ therapy change rate in the Ab testing arm. It also provided $61 \%$ power to demonstrate a significant difference, at an experiment-wise two-tailed $\alpha$-level of 0.05 between a therapy change rate of $5 \%$ in the usual care arm and $8 \%$ in the Ab testing arm.

The modified intention-to-treat (mITT) population was used for all analyses (usual care arm: at least one post-baseline assessment of change in therapy; Ab testing arm: one BAb/NAb test that preceded an assessment of change in therapy). All analyses were conducted with SAS.

Chi-squared and Fisher's exact tests were used to evaluate the association between the initial $\mathrm{Ab}$ status and the type and reasons for therapy changes, as well as the occurrence of targeted AEs. The $p$ value for the outcome measures was based on the difference between study arms from a multivariate logistic regression model with the following covariates: type of MS, time from the onset of MS symptoms, type of current IFN $\beta$ treatment, and duration of current IFN $\beta$ treatment. The analysis model was adjusted for center size and decider status (multiple versus single). A $p$ value $<0.05$ was deemed significant.

Multivariate models with repeated measures utilised all data from $\mathrm{BAb} / \mathrm{NAb}$ testing and types of therapy change, reasons for therapy change, or AEs assessed at the same visit. Models were adjusted for age, sex, study arm, MS type, current IFN, time from onset of symptoms, and center size. Some parameters were not estimable because of low sample size (therapy change occurred less frequently). The linear trend $p$ value tested for the presence of a linear trend across BAb/NAb groups.

Percentages of $\mathrm{BAb}(+)$ patients within $\mathrm{NAb}$ titre level were calculated at the initial visit using $A b$ status as a four-level categorical variable: (i) $\mathrm{BAb}(-)$, (ii) $\mathrm{BAb}(+) / \mathrm{NAb}$ 
Table 1 Demographic and clinical characteristics of modified intention-to-treat cohort at initial assessment stratified by study arm

\begin{tabular}{|c|c|c|c|}
\hline Characteristic & $\begin{array}{l}\text { Scheduled Ab } \\
\text { testing }\end{array}$ & $\begin{array}{l}\text { Usual } \\
\text { care }\end{array}$ & $\begin{array}{c}\text { All } \\
\text { patients }\end{array}$ \\
\hline Patients, n (\%)* & $653(53.1)$ & $577(46.9)$ & $1230(100)$ \\
\hline Age in years, mean $\pm S D$ & $45.3 \pm 10.6$ & $\begin{array}{c}45 \cdot 1 \pm \\
9.8\end{array}$ & $45.2 \pm 10.2$ \\
\hline Caucasians, n (\%) ${ }^{\dagger}$ & $551(84.4)$ & $495(85.8)$ & $1046(85.0)$ \\
\hline Females, n (\%) ${ }^{\dagger}$ & $499(76.4)$ & 441 (76.4) & $940(76.4)$ \\
\hline $\begin{array}{l}\text { Years from onset of } \\
\text { symptoms, mean } \pm S D\end{array}$ & $8.5 \pm 7.7$ & $8 \cdot 5 \pm 7.8$ & $8.5 \pm 7.8$ \\
\hline \multicolumn{4}{|l|}{$\begin{array}{l}\text { Initial high-dose } \\
\text { IFN } \beta, \mathrm{n}(\%)^{\dagger}\end{array}$} \\
\hline IFNß-1a & $467(71.5)$ & $411(71.2)$ & $878(71.4)$ \\
\hline IFN $\beta-1 b$ & $186(28.5)$ & $166(28.8)$ & 352 (28.6) \\
\hline \multicolumn{4}{|l|}{$\begin{array}{l}\text { Time on IFN } \beta \text { in } \\
\text { years, mean }\end{array}$} \\
\hline IFNß-1a & 2.2 & 2.3 & 2.3 \\
\hline IFN $\beta-1 b$ & 2.3 & 2.2 & 2.3 \\
\hline \multicolumn{4}{|l|}{$\begin{array}{l}\text { Initial antibody } \\
\text { status, } \mathrm{n}(\%)^{\ddagger}\end{array}$} \\
\hline $\mathrm{BAb}(+)$ & $264(40.4)$ & $231(40.1)$ & $495(40.3)$ \\
\hline $\mathrm{Bab}(+) \mathrm{NAb}(+)$ & $118(18.1)$ & $114(19.8)$ & 232 (18.9) \\
\hline \multicolumn{4}{|l|}{$\begin{array}{l}\text { NAb titre in NAb (+) } \\
\text { patients, } n(\%)\end{array}$} \\
\hline Moderate & $39(6.0)$ & $43(7.5)$ & $82(6.7)$ \\
\hline High & 79 (12.1) & 71 (12.3) & $150(12.2)$ \\
\hline
\end{tabular}

*Percentage of mITT cohort.

${ }^{\dagger}$ Percentage of study arm.

₹Total $N=1229$. Analysis excludes one patient who had no valid laboratory result at Visit 1.

$A b$, antibody; BAb, serum binding antibodies; IFN $\beta$, interferon beta; NAb, serum neutralizing antibodies; SD, standard deviation.

(-), (iii) $\mathrm{BAb}(+) / \mathrm{NAb}(+)$ moderate (Ab titre $\geq 20$ to $<100$ units, and (iv) $\mathrm{BAb}(+) / \mathrm{NAb}(+)$ high (Ab titre $\geq 100$ units).

Cross tabulations were generated showing the number of patients within each category at the initial and final study visits. McNemar's test was used to test the equality of marginal frequencies (BAb[-] versus all combined $\mathrm{BAb}[+]$ results).

Receiver-operating characteristic (ROC) analysis was plotted, with each point indicating sensitivity and specificity for a particular BAb value in predicting NAb positivity [13], and the predictive ability was estimated by calculating the area under the curve (AUC).

A linear regression model and a Kendall's tau were used to analyze the relationship between BAb and NAb. The $R^{2}$ represented the amount of data variability that could be explained by the model and was similar to a correlation coefficient.
Table 2 Types of management changes and reasons for changes by arm for modified intention-to-treat cohort

\begin{tabular}{|c|c|c|c|}
\hline Category & $\begin{array}{l}\text { Scheduled Ab } \\
\text { testing }(n=653)\end{array}$ & $\begin{array}{l}\text { Usual care } \\
(n=577)\end{array}$ & $p$-value \\
\hline $\begin{array}{l}\text { Number of patients who had } \\
\text { a therapy change, } \mathrm{n}(\%)\end{array}$ & $128(19.6)$ & $81(14.0)$ & 0.004 \\
\hline \multicolumn{4}{|l|}{$\begin{array}{l}\text { Type of therapy change, } \\
n(\%)^{*, t}\end{array}$} \\
\hline Start glatiramer acetate & $47(7.2)$ & $17(2.9)$ & 0.002 \\
\hline $\begin{array}{l}\geq 1 \text { courses of corticosteroids } \\
\text { for relapse }\end{array}$ & $67(10.3)$ & $27(4.7)$ & 0.001 \\
\hline \multicolumn{4}{|l|}{$\begin{array}{l}\text { Reasons for therapy/ } \\
\text { management change, } \\
\text { n }(\%)^{\ddagger, \S}\end{array}$} \\
\hline NAb result & $45(6.9)$ & $3(0.5)$ & $<0.0001$ \\
\hline Clinical composite & $144(22.1)$ & $96(16.6)$ & 0.011 \\
\hline Other & $35(5.4)$ & $16(2.8)$ & 0.011 \\
\hline
\end{tabular}

*Patients reporting a change in type of therapy one or more times were counted once at the earliest occurrence for the same category.

${ }^{\dagger}$ Types of therapy change that were not significantly different between arms included: stop or increase or decrease current interferon beta (IFN $\beta$ ), start new IFN $\beta$, start other immunotherapy, change symptomatic therapy, and closer vigilance. ${ }^{\mp}$ Multiple reasons could be reported for one therapy change; however, patients reporting the same reason more than once were only counted once at the earliest occurrence.

${ }^{\S}$ Reasons for therapy change that were not significantly different between arms included: clinical worsening of multiple sclerosis, magnetic resonance imaging change, side effect, other concurrent illness/adverse event, laboratory abnormality, and administrative/logistical reason.

"Either clinical worsening or change on magnetic resonance imaging.

$A b$, antibody; NAb, serum neutralizing antibody.

\section{Results}

The first patient was enrolled in July 2006, and the last assessment was made in April 2009. Disposition of patients is shown in Figure 1. A total of 1230 patients composed the mITT population. (One patient was excluded from the post-hoc analyses because of the absence of valid laboratory data at Visit 1.)

Table 1 shows the demographic and clinical characteristics of patients at the initial assessment stratified by study arm. At initial assessment, $18.9 \%$ of the mITT patients were $\mathrm{NAb}(+)$. Of the patients who were $\mathrm{BAb}(+)$, $46.9 \%$ were also $\mathrm{NAb}(+)$ at the initial assessment. No relationship was observed between initial antibody status and age, sex, or duration of illness (data not shown).

Subjects who received IFN $\beta$-1a $44 \mu$ g t.i.w. had a higher proportion of positive NAb test results in both the NAb testing (14.2\% [93/653]) and usual care (11.8\% [68/577]) arms than patients receiving IFN $\beta-1 \mathrm{~b} 250 \mu \mathrm{g}$ on alternate days (5.2\% [34/653] and 7.3\% [42/577], respectively) or IFN $\beta$ 1a $22 \mu$ g ti.w. (0.5\% [3/653] and 0.9\% [5/577], respectively).

\section{Primary and secondary outcome measures}

Types of and reasons for therapy change in the Ab testing versus usual care arm

The proportion of patients who had a change in IFN $\beta$ therapy or management during the 12-month follow-up 
Table 3 BAb/NAb status in patients with both initial and last assessments

\begin{tabular}{lcccc}
\hline & \multicolumn{4}{c}{ Status at last assessment, $\mathbf{n}(\%)$} \\
\cline { 2 - 5 } Status at initial assessment & $\mathbf{B A b}(-)$ & $\mathbf{B A b}(+) / \mathbf{N A b}(-)$ & $\mathbf{B A b}(+) / \mathbf{N A b}(+)$ moderate & BAb(+)/NAb(+) high \\
\hline $\mathrm{BAb}(-), \mathrm{n}=624$ & $570(91.4)^{*}$ & $40(6.4)^{\ddagger}$ & $7(1.1)^{\ddagger}$ & $7(1.1)^{\ddagger}$ \\
$\mathrm{BAb}(+) / \mathrm{NAb}(-), \mathrm{n}=222$ & $81(36.5)^{\dagger}$ & $122(55.0)^{*}$ & $16(7.2)^{\ddagger}$ & $3(1.4)^{\ddagger}$ \\
$\mathrm{BAb}(+) / \mathrm{NAb}(+)$ moderate, $\mathrm{n}=69$ & $14(20.3)^{\dagger}$ & $14(20.3)^{\dagger}$ & $29(42.0)^{*}$ & $12(17.4)^{\ddagger}$ \\
$\mathrm{BAb}(+) / \mathrm{NAb}(+)$ high, $\mathrm{n}=130$ & $9(6.9)^{\dagger}$ & $2(1.5)^{\dagger}$ & $9(6.9)^{\dagger}$ & $110(84.6)^{*}$ \\
\hline
\end{tabular}

*Patients who maintained titre.

†Decrease in titre.

\#Increase in titre.

$\mathrm{BAb}(-)$, serum binding antibody negative; $\mathrm{NAb}(-)$, serum neutralizing antibody negative.

NOTE: BAb(-), <4 units; $\mathrm{BAb}(+),>4$ units; NAb(-), $<20$ units; NAb(+) moderate, $\geq 20$ units to $<100$ units; NAb(+) high, $\geq 100$ units.

$\mathrm{BAb}$, serum binding antibodies; NAb, serum neutralizing antibodies.

was significantly higher in the $\mathrm{Ab}$ testing arm than in the usual care arm $(p=0.004$; Table 2).

The main therapy/management changes for both arms are shown in Table 2 . Significantly more patients in the Ab testing arm initiated glatiramer acetate or $\geq 1$ course(s) of corticosteroids for relapse than in the usual care arm ( $p=0.002$ or $p<0.001$, respectively; Table 2 ).

$\mathrm{Ab}$ testing was more often the reason for therapy change in the Ab testing arm than in the usual care arm $(p<0.0001$; Table 2). Clinical composite (either MS clinical worsening or MRI changes) was the most frequent reason for therapy change in both arms and more frequently indicated in the Ab testing than in the usual care arm $(p=0.011$; Table 2). Other reasons for therapy change (i.e., patient decision, $\mathrm{AE}$, pregnancy, financial, and desire to switch therapy) were infrequently reported in the study but were reported more frequently in the $\mathrm{Ab}$ testing arm than in the usual care arm $(p<0.011$; Table 2).

\section{Exploratory outcome measures} Relationship between $B A b$ and NAb status

The BAb and NAb status for the mITT population (both patient groups) with both initial and final assessments are reported in Table 3 . The majority of patients who were $\mathrm{BAb}(-)$ at study initiation remained $\mathrm{BAb}(-)$ at the final assessment $(570 / 624 ; 91 \%)$, while a quarter of patients $(104 / 421 ; 24.7 \%)$ who were $\mathrm{BAb}(+)$ at the initial assessment were $\mathrm{BAb}(-)$ at the final assessment.

$\mathrm{BAb}$ and NAb titre levels fluctuated for approximately $50 \%$ of patients who were $\mathrm{NAb}(+)$ with moderate titres. More than $90 \%$ of $\mathrm{NAb}(+)$ patients who had high titres $(\geq 100$ units) at study initiation were $\mathrm{NAb}(+)$ at the last assessment (Table 3).

AUC by ROC analysis was 0.86 , indicating a strong correlation between BAb level and $\mathrm{NAb}(-)$ and $\mathrm{NAb}(+)$ status. Logistic regression analysis demonstrated BAb titres were a significant predictor of NAb positivity $(p=0.0012)$, with an odds ratio of 1.03 (95\% confidence interval: 1.01

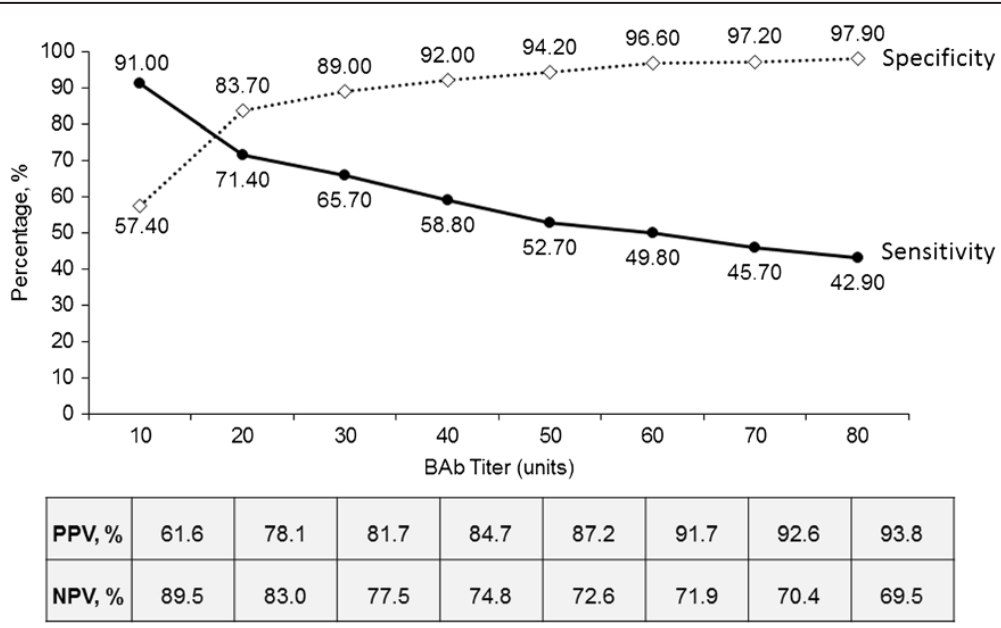

Figure 2 Receiver operating characteristic curve and test characteristics for selected serum binding antibody (BAb) titres of predicting serum neutralizing antibody (NAb) positivity. Positive predicted value (PPV) is the probability that the patient is truly NAb-positive (NAb $>20$ ), given that the patient has a positive test based on BAb titre (BAb > chosen threshold). Negative predicted value (NPV) is the probability that the patient is truly NAb-negative (NAb $<20$ ), given that the patient has a negative test based on BAb titre (BAb < chosen threshold). 
to 1.04). ROC analysis with test characteristics for selected $\mathrm{BAb}$ titres is illustrated in Figure 2. For example, when $\mathrm{BAb}=20$ units, the probability that the patient is truly $\mathrm{NAb}(+)$ is $78.1 \%$.

An increased mean BAb level was observed with higher NAb titre categories. The correlation between $\mathrm{BAb}$ and NAb test results in the mITT population was statistically significant $(p<0.0001)$ using both Kendall's tau $(\tau=0.54)$ and standard linear regression $\left(R^{2}=0.5264\right)$, indicating a significant positive relationship between BAb and NAb test results (Figure 3).

\section{Relationship between initial BAb and NAb status and type of/reason for therapy change}

Antibody status and type of or reason for therapy change for the Ab testing and usual care arms are shown in Table 4. In the Ab testing arm, patients with an initial status of $\mathrm{NAb}(+)$ or $\mathrm{BAb}(+)$ were significantly more likely to change therapy $(p<0.0001$ and $p=0.0054$, respectively). Glatiramer acetate $(p<0.0001$ for both) and closer vigilance were the most frequent types of therapy change in these patients $(p<0.0001$ and $p=0.0004$, respectively) (Table 4).

Reasons for therapy change for patients with positive $\mathrm{Ab}$ status within the $\mathrm{Ab}$ testing arm include NAb results $(p<0.0001$ for both arms), MS clinical worsening $(p=0.0071$ and $p=0.0222$ for $\mathrm{NAb}[+]$ and $\mathrm{BAb}$ $[+]$, respectively), MRI changes $(p<0.0001$ and $p=0$. 0019 , respectively), or clinical composite $(p<0.0001$ and $p=0.0031$, respectively; Table 4$)$.

In the usual care arm, initial $\mathrm{BAb}(+)$ status had a significant effect on the overall number of patients who

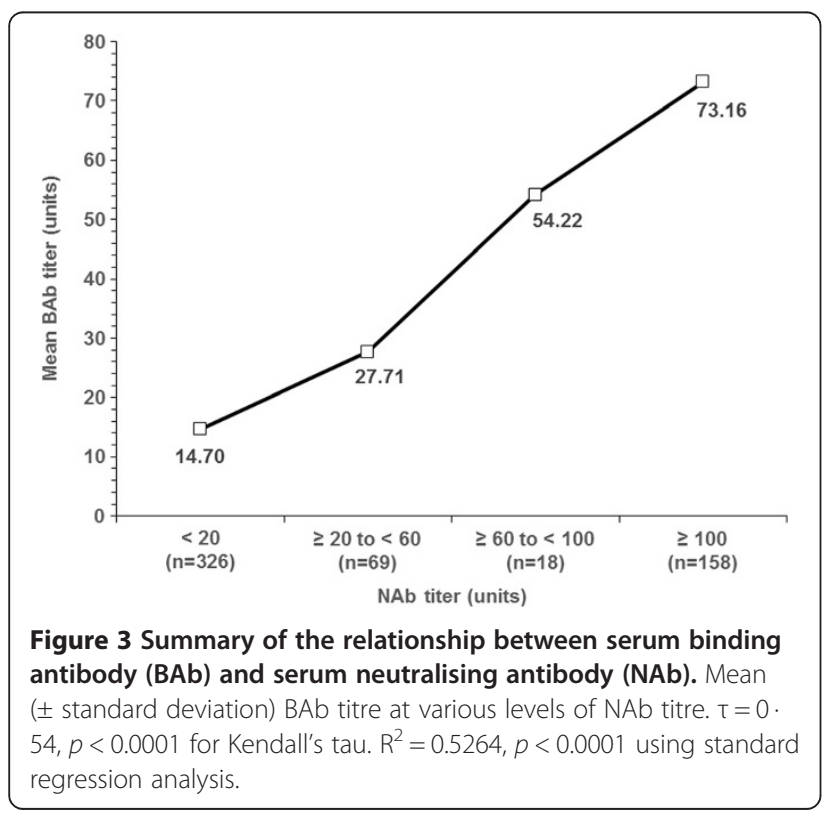

had a change in therapy $(p=0.0241$; Table 4$)$. A greater proportion of patients with initial $\mathrm{BAb}(+)$ versus $\mathrm{BAb}(-)$ status cited clinical worsening of MS $(p=0.0034)$ or clinical composite score $(p=0.0043)$ as their reason for therapy change. $\mathrm{BAb}(+)$ patients receiving usual care had more clinical relapses but not more MRI activity compared with $\mathrm{BAb}(-)$ patients receiving usual care, while $\mathrm{BAb}(+)$ patients had both more MRI activity and clinical relapses than $\mathrm{BAb}(-)$ patients when they received scheduled testing. $\mathrm{NAb}(+)$ patients had significantly more MRI activity $(p=0.0001)$ and clinical relapses $(p=0.0071)$ compared with $\mathrm{NAb}(-)$ patients when they received scheduled testing, but this was not observed when they received usual care (Table 4).

\section{Targeted adverse events}

When analyzing both arms together, patients with NAb titres $\geq 100$ units were significantly less likely to have flu-like symptoms $(p<0.001)$, injection-site reactions $(p<0.001)$, and depression $(p<0.046)$ compared with BAb $(-)$ patients (Table 5). Moreover, patients with an NAb titre of 20 to 100 units were significantly less likely to have flu-like symptoms $(p<0.005)$ or injection-site reactions $(p=0.003)$ compared with $\mathrm{BAb}(-)$ patients (Table 5). The individual treatment arms had similar findings (data not shown).

There was a significant inverse linear correlation between NAb titre level and flu-like symptoms $(p<0.001)$ and injection-site reactions $(p<0.001)$ (Table 5$)$. The findings for both arms combined (mITT cohort) were similar to the individual treatment arms, except that there was a trend for depression associated with NAb titre level when both arms were combined.

\section{Discussion}

In the present study, knowledge of $\mathrm{Ab}$ test results had an impact on therapy or management choices. More patients in the Ab testing arm had therapy changes than in the usual care arm during the 12-month follow-up. It is important to note that this was a relatively large study with a high rate of completion. Further, all time points were analyzed in both arms, and the endpoint of change in therapy by the 12-month time point was statistically significant. Thus, it is unlikely that the mITT criteria for the different patient cohorts would have had a significant effect on the results presented.

Our findings are consistent with those of a recently published study conducted to determine whether early $\mathrm{BAb}$ titres could predict NAb development. In that study $78.9 \%$ of 164 patients receiving de novo IFN- $\beta$ treatment were $\mathrm{BAb}(+)$ after 3 months. The investigators found that $\mathrm{BAb}$ titres $\geq 1: 2400$ at 3 months predicted NAb 
Table 4 Relationship between initial antibody status and types of and reasons for therapy change for the scheduled antibody testing arm and the usual care arm

\begin{tabular}{|c|c|c|c|c|c|c|}
\hline \multirow[t]{2}{*}{ Outcome } & \multicolumn{6}{|c|}{ Antibody status } \\
\hline & BAb(-) & $\mathrm{BAb}(+)$ & $p$-value & $\mathrm{NAb}(-)$ & $\mathrm{NAb}(+)$ & $p$-value \\
\hline Scheduled $A b$ testing arm, $n$ & 387 & 266 & & 148 & 118 & \\
\hline Patients who changed therapy, n (\%)* & $62(16.0)$ & $66(24.8)$ & 0.0054 & $18(12.2)$ & $48(40.7)$ & $<0.0001$ \\
\hline \multicolumn{7}{|l|}{ Type of therapy change ${ }^{\dagger}$} \\
\hline Start glatiramer acetate & $10(2.6)$ & $37(13.9)$ & $<0.0001$ & $6(4.1)$ & $31(26.3)$ & $<0.0001$ \\
\hline New/change in symptomatic therapy & $10(2.6)$ & $10(3.8)$ & 0.3917 & $2(1.4)$ & $8(6.8)$ & 0.0096 \\
\hline Closer vigilance & $13(3.4)$ & $27(10.2)$ & 0.0004 & $8(5.4)$ & $19(16.1)$ & $<0.0001$ \\
\hline \multicolumn{7}{|l|}{ Reason for change ${ }^{*, \dagger}$} \\
\hline NAb result & $2(0.5)$ & $43(16.2)$ & $<0.0001$ & $1(0.7)$ & $42(35.6)$ & $<0.0001$ \\
\hline MS clinical worsening & $55(14.2)$ & $56(21.1)$ & 0.0222 & $26(17.6)$ & $30(25.4)$ & 0.0071 \\
\hline MRI changes & $11(2.8)$ & $22(8.3)$ & 0.0019 & $6(4.1)$ & $16(13.6)$ & $<0.0001$ \\
\hline Clinical composite $^{\ddagger}$ & $60(15.5)$ & $66(24.8)$ & 0.0031 & $27(18.2)$ & $39(33.1)$ & $<0.0001$ \\
\hline Usual care arm, $\mathbf{n}$ & 343 & 233 & & 119 & 114 & \\
\hline Patients who changed therapy, n (\%)* & $39(11.4)$ & $42(18.0)$ & 0.0241 & $24(20.2)$ & $18(15.8)$ & 0.5537 \\
\hline \multicolumn{7}{|l|}{ Type of therapy change $^{\dagger}$} \\
\hline Start glatiramer acetate & $4(1.2)$ & $13(5.6)$ & 0.0021 & $6(5.0)$ & $7(6.1)$ & 0.0247 \\
\hline New/change in symptomatic therapy & $8(2.3)$ & $5(2.2)$ & 0.8825 & $4(3.4)$ & $1(0.9)$ & 0.2681 \\
\hline Closer vigilance & $14(4.1)$ & $11(4.7)$ & 0.7117 & $5(4.2)$ & $6(5.3)$ & 0.5892 \\
\hline \multicolumn{7}{|l|}{ Reason for change $e^{*, \dagger}$} \\
\hline NAb result & $1(0.3)$ & $2(0.9)$ & 0.5687 & $0(0.0)$ & $2(1.8)$ & 0.1015 \\
\hline MS clinical worsening & $32(9.3)$ & $41(17.6)$ & 0.0034 & $21(17.6)$ & $20(17.5)$ & 0.0809 \\
\hline MRI changes & $13(3.8)$ & $10(4.3)$ & 0.7628 & $6(5.0)$ & $4(3.5)$ & 0.7681 \\
\hline Clinical composite ${ }^{\neq}$ & $36(10.5)$ & $44(18.9)$ & 0.0043 & $23(19.3)$ & $21(18.4)$ & 0.1182 \\
\hline
\end{tabular}

*Percentage based on total number of patients in category.

${ }^{\dagger}$ Types of therapy change that were not significantly different for either arm included: current interferon beta (IFN $\beta$ ), increase IFN $\beta$, decrease IFN $\beta$, start new IFN $\beta$, start other immunotherapy, and initiate corticosteroids for relapse. Reasons for therapy change that were not significantly different for either arm included side effect, other concurrent illness/adverse event, laboratory abnormality, administrative/logistical reason, and other.

FEither clinical worsening or MRI change.

$\mathrm{Ab}$, antibody; BAb, serum binding antibody; MRI, magnetic resonance imaging; MS, multiple sclerosis; NAb, serum neutralizing antibody.

evolution with a sensitivity of $74.7 \%$ and a specificity of 98.5\% [14].

Consistent with previous study findings [1], our study found NAb positivity to vary by formulation and dosage frequency of IFN $\beta$ treatment. Patients who received IFN $\beta$-1a $44 \mu \mathrm{g}$ subcutaneously t.i.w. had higher positive $\mathrm{NAb}$ test results than patients on IFN $\beta-1 \mathrm{~b} 250 \mu \mathrm{g}$ on alternate days or IFN $\beta-1 \mathrm{a} 22$ t.i.w. This finding suggests that consideration of the rate at which $\mathrm{Ab}$ titres develop for different treatment regimens is warranted.

As with $\mathrm{NAb}(+)$ status, $\mathrm{BAb}(+)$ status was shown to predict treatment patterns. An initial $\mathrm{BAb}(+)$ status had a significant effect on the overall number of patients changing therapy and on clinical manifestations of MS. The frequency and risk of AEs typically associated with IFN therapy were lower in subjects with higher NAb titres, which suggests that the neutralising effect of the antibodies on IFN $\beta$ may have caused a reduction in these events. This association between NAb titre and AEs is supported by previous reports.

Overall, data support the use of BAb titres from $A b$ testing to predict NAb positivity. We found that if a patient had $\mathrm{BAb}>50$ units, there was a very high probability that the patient was $\mathrm{NAb}(+)$, and treatment decisions could be adequately based on this information.

Although there are no widely accepted guidelines on $\mathrm{BAb}$ test-driven management, a titre cutoff point for $\mathrm{BAb}$ could be used to predict the risk of $\mathrm{NAb}(+)$ and the necessity for medication change [15]. A recent study [16] suggested using a high BAb titre as a cutoff point for IFN therapy, because of a strong correlation between $\mathrm{NAb}$ and $\mathrm{BAb}$ in this group. Moreover, in patients with low titres, the study suggested refining the $B A b$ assay by incorporating an MxA induction assay to 
Table 5 Relationship between antibody level throughout the study and targeted side effects for both arms combined (mITT cohort) and individual arms*

\begin{tabular}{|c|c|c|c|c|c|}
\hline Category & Antibody level (units) & Odds ratio & $\begin{array}{c}95 \% \text { confidence } \\
\text { interval }\end{array}$ & $\begin{array}{c}p \text {-value } \\
\text { (odds ratio) }\end{array}$ & $\begin{array}{c}p \text {-value } \\
\text { (linear trend) }\end{array}$ \\
\hline \multicolumn{6}{|c|}{ Targeted side effects for both arms combined (mITT cohort) } \\
\hline \multirow[t]{4}{*}{ Flu-like symptoms in past month } & $N A b \geq 100$ & 0.33 & $(0.22,0.48)$ & $<0.001$ & $<0.001$ \\
\hline & NAb 20 to $<100$ & 0.41 & $(0.28,0.60)$ & $<0.005$ & \\
\hline & $\mathrm{NAb}<20$ & 0.92 & $(0.71,1.18)$ & 0.50 & \\
\hline & $\mathrm{BAb}(-)$ & (Ref) & - & - & \\
\hline \multirow[t]{4}{*}{ Injection site reactions in past month } & $N A b \geq 100$ & 0.34 & $(0.25,0.45)$ & $<0.001$ & $<0.001$ \\
\hline & NAb 20 to $<100$ & 0.59 & $(0.41,0.83)$ & 0.003 & \\
\hline & $\mathrm{NAb}<20$ & 0.79 & $(0.62,1.01)$ & 0.062 & \\
\hline & $\mathrm{BAb}(-)$ & (Ref) & - & - & \\
\hline \multirow[t]{4}{*}{ Depression in past month } & $N A b \geq 100$ & 0.70 & $(0.50,0.99)$ & 0.046 & 0.083 \\
\hline & NAb 20 to $<100$ & 0.99 & $(0.68,1.44)$ & 0.95 & \\
\hline & $N A b<20$ & 0.93 & $(0.71,1.22)$ & 0.60 & \\
\hline & $\mathrm{BAb}(-)$ & (Ref) & - & - & \\
\hline
\end{tabular}

* Repeated measures multivariable model utilized all data for which BAb/NAb testing and adverse events were assessed at the same visit. Models were adjusted for age, sex, study arm, multiple sclerosis type, current interferon, time from onset of symptoms, and centre size. Patients who were BAb(-) served as the referent, with an odds ratio calculated to represent the odds that a particular NAb level group would experience the event, compared with the referent. The linear trend $p$-value tested for the presence of a linear trend across BAb/NAb groups. Targeted events that did not show a statistically significant effect are not included in the table (seizures, menstrual irregularities, abnormal liver function tests, cytopenia, and decreased hemoglobin).

$\mathrm{BAb}$, serum binding antibody; mITT, modified intention-to-treat; NAb, serum neutralizing antibody.

establish whether the bioavailability of IFN $\beta$ is preserved. Results from our ROC analysis indicate there is a very high probability of patients with $\mathrm{BAb}>50$ units (specificity $\geq 94 \%$ ) and even as low as 20 units (specificity $>80 \%$ ) being $\mathrm{NAb}(+)$, which could mean that lower BAb titre cutoff points may be sufficient to guide treatment decisions.

\section{Limitations of study}

One shortcoming of this study is the absence of paired clinical response data. Although the study was not designed to follow this outcome, the correlation between clinical worsening and $\mathrm{NAb}(+)$ response in patients on IFN $\beta$ therapy is well established $[4,17]$. Another consideration when interpreting the results is the imbalance in clinic visits that the $\mathrm{Ab}$ testing arm would have received versus the usual treatment arm, which could have affected management choices irrespective of $\mathrm{Ab}$ results. Moreover, it could be argued that the study duration was inadequate. However, the study duration of 12 months represents real-world practice and was sufficient to confirm both the relative stability of $\mathrm{NAb} / \mathrm{BAb}$ levels and the strong correlation of BAb titres with NAb positivity. In addition, patients were required to complete 1-4 years of therapy before study entry, the time frame of greatest importance in NAb development.
It should be noted that our study involved formulations of IFN $\beta$-1a currently available in the US, as used in the EVIDENCE and REGARD studies, Rebif, 22 or $44 \mu \mathrm{g}$ t.i.w., and Avonex , $30 \mu \mathrm{g}$ weekly (considered low dose); a newer formulation of Rebif has since been introduced in other countries with the aim of inducing less NAb positivity [18].

\section{Conclusion}

Testing for NAb is an important aspect of MS management in terms of predicting treatment response to IFN $\beta$. Using BAb testing before screening for NAb titres, and defining a cutoff point for the BAb titres at which to discontinue IFN $\beta$ therapy, may reduce the necessity for the more expensive NAb testing assays.

\section{Abbreviations}

Ab: Antibody; AEs: Adverse events; AUC: Area under the curve; BAb: Serum binding antibodies; EFNS: European Federation of Neurologic Societies; IFNß: Interferon- $\beta$; IgG: Immunoglobulin G; mITT: Modified intention-to-treat; MRI: Magnetic resonance imaging; MS: Multiple sclerosis; NAb: Neutralizing antibodies; ROC: Receiver-operating characteristic; t.i.w: Three-times weekly.

\section{Competing interests}

EF has been a consultant for Acorda Pharmaceuticals, Bayer, Biogen Idec, EMD Serono, Genzyme, Novartis, Opexa, Sanofi-Aventis, and Teva Pharmaceuticals. EF has also been a clinical investigator for the following: Avanir, Biogen Idec, EMD Serono, GlaxoSmithKline, Teva Pharmaceuticals, Novartis, Ono, Sanofi-Aventis, Eli Lilly, and Roche Genentech. BG has received honoraria for speaking from Bayer, 
Biogen Idec, Genzyme-Sanofi, Novartis, and Teva Pharmaceuticals. CM reports consultancy/lectureship with Bayer, Biogen Idec, EMD Serono, Novartis, Teva Pharmaceuticals, and Wyeth Pharmaceuticals. CM has received grants from Bayer, Biogen Idec, EMD Serono, Novartis, and Teva Pharmaceuticals. RM has received honoraria for consulting, speaking, and teaching from Teva Pharmaceuticals, Biogen Idec, Genzyme, and Acorda Therapeutics. RM has also received grant and research support from Teva Pharmaceutials, Biogen Idec, Novartis, and Acorda Therapeutics. RM participated on advisory committees or review panels for Teva Pharmaceuticals, Biogen Idec, Novartis, and Genzyme. ADG has received honoraria for consulting services from Acorda Therapeutics, Avanir, Biogen Idec, EMD Serono, Genzyme-Sanofi, Novartis, Pfizer, and Teva Pharmaceuticals and financial support for research activities from Acorda Therapeutics, Biogen Idec, EMD Serono, Genzyme, Novartis, Ono, Sun Pharma, Takeda, and Teva Pharmaceuticals. SJG, PL, and JNC are employees of Teva Pharmaceutical Industries, Kansas City, MO, USA.

\section{Authors' contributions}

All authors contributed equally to the development of this manuscript. All authors read and approved the final manuscript.

\section{Acknowledgements}

Teva Pharmaceuticals (Petach Tikva, Israel) funded the study and participated in study design, management, and manuscript preparation. ICON Clinical Research (San Francisco, CA) conducted the statistical analysis, which was funded by Teva Pharmaceuticals. The authors had full access to all data and analytical results and had final responsibility for the decision to submit the manuscript for publication.

The authors would like to thank Ruth Sussman (Chameleon Communications International, New York, NY, USA) and Heather Oliff (Science Consulting Group, North Tustin, CA, USA) for assistance with manuscript development and Lawrence Rasouliyan, MPH (ICON Late Phase and Outcomes Research, San Francisco, CA, USA) for statistical support.

\section{Author details}

${ }^{1}$ Central Texas Neurology Consultants, 16040 Park Valley Drive, Suite 100, Round Rock, TX 78681, USA. ²West County MS Center, Mercy Hospital, St. Louis, MO, USA. ${ }^{3}$ MS Center, Neurology Department, University of Pennsylvania School of Medicine, Philadelphia, PA, USA. ${ }^{4}$ Multiple Sclerosis Clinic of Colorado, Lone Tree, CO, USA. ${ }^{5}$ Neurology Department, University of Rochester School of Medicine and Dentistry, Rochester, NY, USA. ${ }^{6}$ Teva Pharmaceuticals, Kansas City, MO, USA.

Received: 5 November 2013 Accepted: 28 March 2014 Published: 4 April 2014

\section{References}

1. Ross C, Clemmesen K, Svenson M, Sørensen PS, Koch-Henriksen N, Skovgaard GL, Bendtzen K: Immunogenicity of interferon-beta in multiple sclerosis patients: influence of preparation, dosage, dose frequency, and route of administration. Danish Multiple Sclerosis Study Group. Ann Neurol 2000, 48:706-712

2. Creeke PI, Farrell RA: Clinical testing for neutralizing antibodies to interferon- $\beta$ in multiple sclerosis. Ther Adv Neurol Disord 2013, 6:3-17.

3. Sørensen PS, Deisenhammer F, Duda P, Hohlfeld R, Myhr KM, Palace J, Polman C, Pozzilli C, Ross C, EFNS Task Force on Anti-IFN-beta Antibodies in Multiple Sclerosis: Guidelines on use of anti-IFN-beta antibody measurements in multiple sclerosis: report of an EFNS Task Force on IFN-beta antibodies in multiple sclerosis. Eur J Neurol 2005, 12:817-827.

4. Fox EJ, Vartanian TK, Zamvil SS: The immunogenicity of disease-modifying therapies for multiple sclerosis: clinical implications for neurologists. Neurologist 2007, 13:355-362.

5. Paolicelli D, D'Onghia M, Pellegrini F, Direnzo V, laffaldano P, Lavolpe V, Trojano M: The impact of neutralizing antibodies on the risk of disease worsening in interferon $\beta$-treated relapsing multiple sclerosis: a 5 year post-marketing study. J Neurol 2013, 260:1562-1568.

6. Sorensen PS, Ross C, Clemmesen KM, Bendtzen K, Frederiksen $J$, Jensen $K$, Kristensen O, Petersen T, Rasmussen S, Ravnborg M, Stenager E, Koch-Henriksen N, Danish Multiple Sclerosis Study Group: Clinical importance of neutralising antibodies against interferon beta in patients with relapsing-remitting multiple sclerosis. Lancet 2003, 362:1184-1191.
7. Namaka M, Pollitt-Smith M, Gupta A, Klowak M, Vasconcelos M, Turcotte D, Gong Y, Melanson M: The clinical importance of neutralizing antibodies in relapsing-remitting multiple sclerosis. Curr Med Res Opin 2006, 22:223-239.

8. Goodin DS, Frohman EM, Hurwitz B, O'Connor PW, Oger JJ, Reder AT, Stevens JC: Neutralizing antibodies to interferon beta: assessment of their clinical and radiographic impact: an evidence report of the Therapeutics and Technology Assessment Subcommittee of the American Academy of Neurology. Neurology 2007, 68:977-984.

9. Malucchi S, Sala A, Gilli F, Bottero R, Di Sapio A, Capobianco M, Bertolotto A: Neutralizing antibodies reduce the efficacy of beta IFN during treatment of multiple sclerosis. Neurology 2004, 62:2031-2037.

10. Sorensen PS, Tscherning T, Mathiesen HK, Langkilde AR, Ross C, Ravnborg M, Bendtzen K: Neutralizing antibodies hamper IFN beta bioactivity and treatment effect on MRI in patients with MS. Neurology 2006, 67:1681-1683.

11. Goodin D, Hartung H, O'Connor P, Filippi M, Arnason B, Comi G, Cook S, Jeffery D, Kappos L, Bogumil T, Knappertz V, Sandbrink R, Beckmann K, White R, Petkau J, Pohl C, BEYOND Study Group: Neutralizing antibodies to interferon beta-1b multiple sclerosis: a clinic-radiographic paradox in the BEYOND trial. Mult Scler 2012, 18:181-195.

12. O'Connor P, Devonshire $\mathrm{V}$ : The use of disease-modifying agents in multiple sclerosis-by the Canadian Network of MS Clinics. Can J Neurol Sci 2008, 35:127-132

13. Zweig MH, Campbell G: Receiver-operating characteristic (ROC) plots: a fundamental evaluation tool in clinical medicine. Clin Chem 1993, 39:561-577.

14. Hegen H, Millonig A, Bertolotto A, Comabella M, Giovanonni G, Guger M, Hoelzl M, Khalil M, Killestein J, Lindberg R, Malucchi S, Mehling M, Montalban X, Polman C, Rudzki D, Schautzer F, Sellebjerg F, Sørensen P, Deisenhammer F: Early detection of neutralizing antibodies to interferon-beta in multiple sclerosis patients: binding antibodies predict neutralizing antibody development. Mult Scler 2013. [Epub ahead of print] PMID: 24009164.

15. Polman $\mathrm{CH}$, Bertolotto A, Deisenhammer F, Giovannoni G, Hartung HP, Hemmer B, Killestein J, McFarland HF, Oger J, Pachner AR, Petkau J, Reder AT, Reingold SC, Schellekens H, Sørensen PS: Recommendations for clinical use of data on neutralising antibodies to interferon-beta therapy in multiple sclerosis. Lancet Neurol 2010, 9:740-750.

16. Jensen PE, Sellebjerg F, Søndergaard HB, Sørensen PS: Correlation between anti-interferon- $\beta$ binding and neutralizing antibodies in interferon$\beta$-treated multiple sclerosis patients. Eur J Neurol 2012, 19:1311-1317.

17. Cohen BA, Oger J, Gagnon A, Giovannoni G: The implications of immunogenicity for protein-based multiple sclerosis therapies. J Neurol Sci 2008, 275:7-17.

18. Giovannoni G, Barbarash O, Casset-Semanaz F, King J, Metz L, Pardo G, Simsarian J, Sørensen PS, Stubinski B, Rebif New Formulation Study Group: Safety and immunogenicity of a new formulation of interferon beta-1a (Rebif New Formulation) in a Phase Illb study in patients with relapsing multiple sclerosis: 96-week results. Mult Scler 2009, 15:219-228.

doi:10.1186/1471-2377-14-73

Cite this article as: Fox et al:: The effect of scheduled antibody testing on treatment patterns in interferon-treated patients with multiple sclerosis. BMC Neurology 2014 14:73.

\section{Submit your next manuscript to BioMed Central and take full advantage of:}

- Convenient online submission

- Thorough peer review

- No space constraints or color figure charges

- Immediate publication on acceptance

- Inclusion in PubMed, CAS, Scopus and Google Scholar

- Research which is freely available for redistribution 